\title{
Homogenization of northern U.S. Great Lakes forests due to land use
}

\author{
Lisa A. Schulte - David J. Mladenoff - Thomas R. Crow • \\ Laura C. Merrick · David T. Cleland
}

Published online: 30 May 2007

(C) Springer Science+Business Media B.V. 2007

\section{Erratum to: Landscape Ecol (2007) DOI 10.1007/s10980-007-9095-5}

Replacement of Table 1

The online version of the original article can be found under doi: 10.1007/s10980-007-9095-5

\section{A. Schulte $(\square) \cdot$ L. C. Merrick}

Natural Resource Ecology and Management, Iowa State

University, 339 Science II, Ames, IA 50011, USA

e-mail: 1schulte@iastate.edu

\section{J. Mladenoff}

Forest Ecology and Management, University of

Wisconsin-Madison, Madison, WI 53706, USA

T. R. Crow

USDA Forest Service Wildlife, Fish, Air and Water

Research, Washington, DC 20090, USA

D. T. Cleland

USDA Forest Service Eastern Region and Southern

Research Station, Rhinelander, WI 54501, USA
Table 1 Summary statistics from non-metric multidimensional scaling (NMS) ordination between pre-Euro-American and present periods

\begin{tabular}{|c|c|c|c|}
\hline $\begin{array}{l}\text { Compositional and structural } \\
\text { variables }\end{array}$ & $\begin{array}{l}\text { Axis } 1 \\
\mathrm{r}\end{array}$ & $\begin{array}{l}\text { Axis } 2 \\
\mathrm{r}\end{array}$ & $\begin{array}{l}\text { Axis } 3 \\
\mathrm{r}\end{array}$ \\
\hline Ash (Fraxinus spp.) & 0.23 & -0.38 & -0.59 \\
\hline Aspen (Populus spp.) & -0.13 & 0.06 & -0.81 \\
\hline Basswood (Tilia americana) & 0.36 & -0.47 & -0.33 \\
\hline Beech (Fagus americana) & 0.18 & -0.41 & 0.44 \\
\hline Birch (Betula spp.) & 0.20 & 0.56 & 0.29 \\
\hline Cedar (Thuja occidentalis) & 0.17 & 0.31 & -0.14 \\
\hline Elm (Ulmus spp.) & 0.06 & -0.42 & 0.15 \\
\hline Fir (Abies balsamifera) & 0.15 & 0.58 & -0.47 \\
\hline Hemlock (Tsuga canadensis) & 0.45 & 0.02 & 0.77 \\
\hline Hickory (Carya spp.) & -0.04 & -0.41 & -0.05 \\
\hline Maple (Acer spp.) & 0.77 & -0.35 & -0.17 \\
\hline Oak (Quercus spp.) & -0.23 & -0.63 & -0.30 \\
\hline Pine (Pinus spp.) & -0.80 & 0.05 & 0.42 \\
\hline Spruce (Picea spp.) & -0.21 & 0.76 & -0.24 \\
\hline Tamarack (Larix laricina) & -0.40 & 0.57 & 0.14 \\
\hline Extent open vegetation & -0.08 & -0.33 & -0.86 \\
\hline Percent tree diameters $\geq 51 \mathrm{~cm}$ & -0.09 & -0.22 & 0.51 \\
\hline $\begin{array}{l}\text { Standard deviation in trees } \geq 51 \mathrm{~cm} \\
\text { in diameter }\end{array}$ & 0.02 & 0.18 & 0.51 \\
\hline
\end{tabular}

Data for tree genera are based on relative dominance values 